\title{
Paradoxical air embolism in two horses proven by transthoracic echocardiographic evaluation
}

\author{
Arabella Dutka', Bianca Schwarz ${ }^{1,2}$, Karsten Velde ${ }^{3}$ and Astrid B. M. Rijkenhuizen ${ }^{4}$ \\ 1 Equine Clinic, University of Veterinary Medicine Vienna, Austria \\ 2 Equine Clinic Altforweiler, Überherrn, Germany \\ 3 Equine Clinic, University of Veterinary Medicine Vienna, Austria \\ ${ }^{4}$ Veterinary Clinic Duurstede, Wijk bij Duurstede, the Netherlands
}

Summary: A 19 year old Standardbred gelding and a 5 year old Quarter Horse mare experienced an episode of air embolism due to a dislodged catheter cap. Initial clinical signs in the gelding included tachycardia, arrhythmia, tachypnoea and disorientation. The mare showed agitation, selfmutilation due to severe pruritus, tachycardia with a mill-wheel murmur and tachypnoea. Echocardiography revealed air bubbles in the right and left side of the heart, the pulmonary vessels and the aorta. An anatomical abnormality was excluded by a bubble study, proving a paradoxical air embolism. Both horses received symptomatic treatment and recovered. Air embolism rarely occurs in horses. The extent of air embolism can be shown with echocardiography, which seems to allow a better evaluation of the condition and might be helpful in determining which clinical signs are to be expected.

Keywords: embolism, vein, venous catheter, horse

Citation: Dutka A., Schwarz B., Velde K., Rijkenhuizen A. B. M. (2017) Paradoxical air embolism in two horses proven by transthoracic echocardiographic evaluation. Pferdeheilkunde 33, 329-334; DOI 10.21836/PEM20170402

Correspondence: Prof. Astrid B. M. Rijkenhuizen, Veterinary Clinic Duurstede, Singel 10, 3961 CE Wijk Bij Duurstede, The Netherlands: e-mail: a.rijkenhuizen@gmail.com

\section{Introduction}

Vascular air embolism is rare in horses and it seems mostly to be related to an iatrogenic event of an open IV catheter (Bradbury et al. 2005, Caporelli et al. 2009, Holbrook et al. 2007, Pellegrini-Masini et al. 2009). In humans air embolism is also associated with cerebral surgery in a sitting position, laparoscopy and lung trauma (van Hulst et al. 2003). In contrast to arterial air embolism, which is known to be often fatal with only small amounts of air, in venous air embolism seems to be tolerated more air in most species (van Hulst et al 2003). Clinical signs appear only after slow venous aspiration of large amounts of air or after administering large boluses IV (Adornato et al. 1978), hence venous air embolism seems to be a time and volume dependent incident. Venous air embolism can result in arterial air embolism, if the air cannot be cleared in the venous system, the so-called paradoxical air embolism (Pasternak and Lanier 2017). Until now, the diagnosis in equine cases was based on clinical signs as a result of catheter cap removal (Bradbury et al. 2005, Caporelli et al. 2009, Holbrook et al. 2007, Pellegrini-Masini et al. 2009). In this report, the suspected diagnosis of air embolism in the horse was confirmed by echocardiography.

\section{Case reports}

Case 1

A 19 year old Standardbred gelding was referred to the Equine Hospital of the University of Veterinary Medicine Vienna for evaluation of severe dental problems and was scheduled to undergo tooth extraction and sinus trepanation. Therefore a venous catheter (12 Gauge/80 mm, Intraflon 2) ${ }^{1}$ was placed with the tip towards the heart in the left jugular vein. 20 Minutes after catheter placement the horse was found with an open catheter, missing the catheter cap. The catheter was closed immediately and physical evaluation revealed that the horse was disorientated, sweating and had cold extremities. Furthermore the horse's pulse was not palpable, heart rate was increased to 80 beats/minute and an arrhythmia was noted. The respiratory rate was 60 breaths/minute, other clinical parameters (mucous membranes, capillary refill time, skin elasticity, borborygmy, body temperature) were within normal limits. The horse's vision appeared to be unaffected. Heart auscultation revealed a continuous, millwheel murmur. The horse was placed in the stocks and the echocardiogram (ECG) (Cardiovit CS 100) ${ }^{2}$ showed a supraventricular tachyarrhythmia. The first catheter was removed and a wide bore catheter (8 Gauge/13 cm, Equicath FastFlow) ${ }^{3}$ was placed in the right jugular vein. Polyionic fluids (Vetifundin) ${ }^{4}$ were administered at a rate of approximately $12 \mathrm{~L} / \mathrm{hr}$ until a total volume of $30 \mathrm{~L}$ was reached. Additionally, the horse received $2 \mathrm{~L}$ of colloid fluids (Haes) ${ }^{5}$. Lidocaine (Xylanaest purum $\left.2 \%\right)^{6}$ was administered to treat the cardiac arrhythmia (loading dose of $1.3 \mathrm{mg} / \mathrm{kg}$ for 10 minutes, maintenance of $0.05 \mathrm{mg} / \mathrm{kg} / \mathrm{hr}$ for one hour). Arterial blood gases showed a $\mathrm{pO}_{2}$ of $65 \mathrm{mmHg}$, therefore oxygen was administered intranasally $(15 \mathrm{~L} /$ minute for 40 minutes). Hematology revealed a slight mild leucocytosis $\left(10.5 \times 10^{9} / \mathrm{l}\right.$, reference range $\left.[\mathrm{rr}] 5.0-10.0 \times 10^{9} / \mathrm{l}\right)$, hypokalemia $(2.9 \mathrm{mmol} / \mathrm{l},[\mathrm{rr}] 3.5-4.5 \mathrm{mmol} / \mathrm{l})$, hyperlactaemia (3 mmol/l, [rr] $<2.5 \mathrm{mmol} / \mathrm{l})$ and slightly increased creatine kinase activity $(222 \mathrm{U} / \mathrm{L},[\mathrm{rr}]<190 \mathrm{mmol} / \mathrm{I})$.

\section{Diagnostic Procedure}

Echocardiography was performed (Vivid 3) ${ }^{7}$ with a $3 \mathrm{MHz}$ probe about 40 minutes after the horse was found with the open catheter. Air bubbles, evident as echodense areas, were visible in the right and left atrium, the right and left ventricle, 
the pulmonary artery and the aorta (Figure 1). These findings did not change after discontinuing the IV fluids for 15 minutes. A bubble study was performed immediately to exclude any anatomical abnormalities of the heart. Ten milliliter of the horse's own blood and ten milliliter sterile saline were mixed to create microbubbles and injected through the catheter immediately after shaking (Nanthakumar et al. 2001). The ultrasound probe was positioned on the right side of the chest to provide a standard four chamber view. An increased number of bubbles was found in the right atrium and ventricle, but not on the left side of the heart or in the aorta.

One hour after the horse was found with symptoms, the back muscles appeared hard and swollen, with the left side being more severely affected. A repeated creatine kinase activity measurement revealed $981 \mathrm{U} / \mathrm{l}$. Hyperaemic lotions (Restitutionsfluid) ${ }^{8}$ were applied locally with massage, and the condition improved over the course of the following 12 hours.

Two hours after the incident the gelding appeared to be stable and was put into a stall. At that point heart rate was 56 beats/minute, respiratory rate 20 breaths/minute, lung sounds were slightly harsh, and all other parameters of the clinical examination were within normal limits. The horse was monitored closely and the vital parameters were checked every 20-30 minutes. A holter ECG was performed and the horse was maintained on polyionic fluids at a rate of $2 \mathrm{~L} /$ hour. No changes were noticed on the ECG, except for an increased heart rate, the supraventricular tachyarrhythmia was not be present anymore.

Two hours later heart rate and respiratory rate increased to 76 beats/minute and 32 breath/minute, respectively. The horse showed a shallow breathing pattern and started sweating again. Arterial $\mathrm{pO}_{2}$ was $76 \mathrm{mmHg}$, therefore oxygen was again substituted intra-nasally. Oxygen administration was discontinued after 3 hours as the clinical signs normalised. The heart, pulmonary artery and aorta were re-evaluated ultrasonographically 6 hours after the first assessment and no more air bubbles could be seen.

Seven hours after the initial incidence the gelding suddenly showed signs of focal seizures. Clinical signs included an irregular heart rhythm with a rate of 180-200 beats/minute, a stretched head-neck position, dorsal flexion of the back,

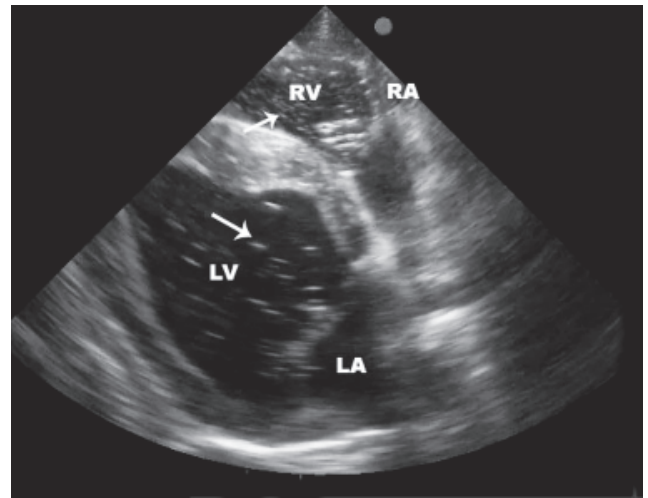

Fig. 1 Echocardiographic findings in case of paradoxical air embolism: air bubbles in both sides of the heart. Case 1. Rightsided four chamber view, $3 \mathrm{MHz}$ probe. RA: right atrium; RV: right ventricle; LA: left atrium; LV: left ventricle; Arrow: air bubbles in left and right ventricle unnaturally wide pupils and severe fasciculations and spasms of the right facial musculature. The horse was treated with $1.5 \mathrm{~g}$ of midazolam (Midazolam Nycomed) ${ }^{9}$ intravenously and responded immediately to the treatment. After a second seizure was observed 20 minutes later, the horse was moved to a padded stall and received $3 \mathrm{~g}$ of phenobarbital (Luminal Injektionslösung) ${ }^{10}$ in $500 \mathrm{ml}$ sterile saline IV slowly over the course of one hour and the neurological signs gradually disappeared.

The horse recovered uneventfully within 12 hours from the onset of clinical signs and fluid therapy was discontinued after 24 hours. All physical parameters remained within normal limits for the reminding hospitalization. Anti-inflammatory medication (Meloxicam (Metacam Injektionslösung) ${ }^{11} 0.6$ $\mathrm{mg} / \mathrm{kg}$ SID IV) was administered immediately after the initial incident and continued for 3 days.

The horse was discharged after 3 days and the owner reported no further abnormalities. Dental surgery was performed after readmission 4 weeks later without any complications. At a follow up phone call 6 month later the owner reported that the horse had recovered to full use as a pleasure horse.

\section{Case 2}

A 5 year old Quarter Horse mare underwent colic surgery for small colon impaction, which was refractory to medical treatment at a private clinic. On day 3 post surgery after an uneventful recovery the mare received an intravenous treatment consisting of antibiotics and NSAIDs (benzylpenicillin/ streptomycin (Veracin-compositum) ${ }^{12} \quad 18000 \mathrm{IU}$ and $10.000 \mathrm{IU} / \mathrm{kg}$ SID IM, gentamicin (Gentamicin 50) ${ }^{13}$ $6.6 \mathrm{mg} / \mathrm{kg}$ SID IV, Flunixin meglumine (Paraflunixin) ${ }^{14}$ $1.1 \mathrm{mg} / \mathrm{kg}$ BID IV) via the catheter $(14$ Gauge/13 cm, Milacath) ${ }^{15}$ in the left jugular vene. 40 Minutes later the horse was found agitated in its box, walking in circles, selfmutilating: biting into the lateral chest wall and kicking its abdomen with the hindlegs. Furthermore she was swishing her tail and showing signs of severe generalised pruritus, mainly biting the lateral chest wall but also the flanks. Physical examination revealed pale pink mucous membranes with a capillary refill time of $3 \mathrm{~s}$; tachypnoea (52 beats/minute) and tachycardia (60 breaths/min). Pulse quality was poor. Heart auscultation revealed a continuous, very loud, millwheel murmur and moderately increased lung sounds on lung auscultation. The laparotomy wound was bleeding due to self-inflicted trauma with the hind legs. The catheter $(14$ Gauge/13cm, Milacath) ${ }^{15}$, which had been placed prior to surgery in the left jugular vein with the tip towards the heart, was noticed to have a defect extension tubing (air was being sucked audibly into the jugular vein) and the extension tubing was replaced.

\section{Diagnostic Procedure}

Approximately 5 minutes after the horse was found with the open catheter echocardiography was performed (MyLab Twice) ${ }^{16}$ with a $2.5 \mathrm{MHz}$ probe. A large amount of air bubbles was visible in the right atrium, right ventricle and pulmonary artery, to a lesser amount air bubbles were also seen in the left atrium, left ventricle and aorta. Large air bubbles, crea- 
ting acoustic shadowing were seen below the tricuspid valve and at the pulmonary valve in the right ventricle.

The horse was sedated with $200 \mathrm{mg}$ xylazine (Xylariem) ${ }^{17}$ IV and received symptomatic treatment with $20 \mathrm{mg} / \mathrm{kg}$ DMSO in $500 \mathrm{ml} \mathrm{NaCl} 0.9 \%$ and $10 \mathrm{~L} \mathrm{NaCl} 0.9 \%$ over one hour. Sedated, the horse stopped the selfmutilating behaviour. 30 Minutes later the millwheel murmur was not audible any more on auscultation. Heart and respiratory rate were still elevated, the remainder of the physical examination was normal, except that the horse showed slight agitation and mild pruritus over the next hours. Two hours later heart and respiratory rate returned within normal limits. No neurological signs were apparent. The horse was closely monitored, kept quiet in its box. Over the next hours the horse remained slightly uncomfortable with mild signs of pruritus, and returned to normal within 24 hours after the incident. A bubble study, as described before, was performed and a connection between left and right side of the heart was excluded (Fig 2 and 3). 24 Hours after the incident the horse developed a periphlebitis characterised by a small $( \pm 2 \mathrm{~cm})$ firm localised swelling at the catheter site and the catheter was taken out immediately and a local treatment was initiated with an anti-inflammatory gel (Compagel). The perithrombophlebitis resolved within one week and the horse was discharged from the hospital 17 days after the episode with air embolism. One year later the

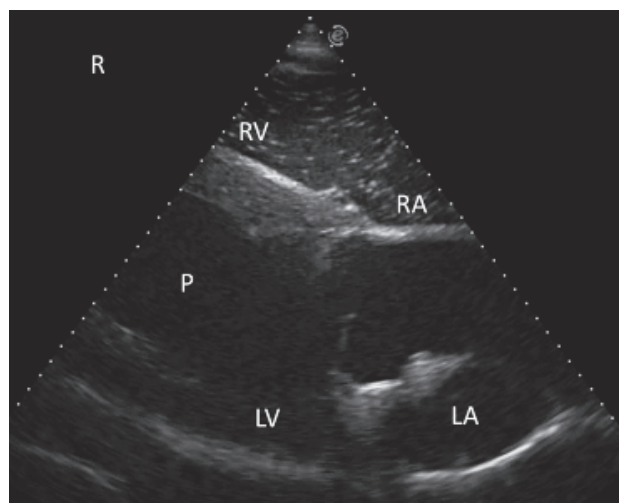

Fig. 2 Echocardiographic findings while performing bubble study. Microbubbles are visible in the right side of the heart (RA, RV) and the right ventricular outflow tract (, but not the left side of the heart. Rightsided four chamber view and right ventricular outflow tract (PA), $2.5 \mathrm{MHz}$ probe. RA: right atrium; $\mathrm{RV}$ : right ventricle; LA: left atrium; $\mathrm{LV}$ : left ventricle

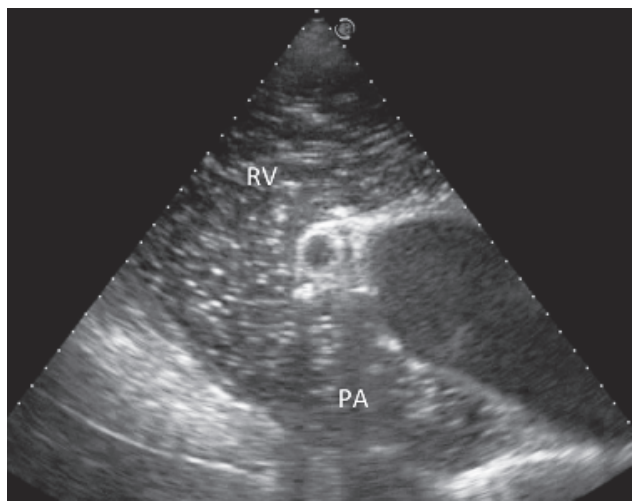

Fig. 3 Echocardiographic findings while performing bubble study. sind im rechtsventrikulären Ausflusstrakt zu sehen (rechter Ventrikel und Pulmonalarterie). Rightsided four chamber view and right ventricular outflow tract (PA), $2.5 \mathrm{MHz}$ probe. RV: right ventricle; PA: pulmonal artery horse is reported to be healthy and is used for pleasure riding.

\section{Discussion}

Vascular air embolism is defined as the entrapment of air from the environment into the vascular system causing systemic effects (Mirski et al. 2007). Clinical signs appear only after slow venous administration of large amounts of air or after administration large boluses IV (Adornato et al. 1978), hence venous air embolism seems to be a time and volume dependent incident. If the air enters the venous system, it usually passes through the right side of the heart and subsequently is cleared in the capillary system of the lung (van Hulst et al. 2003). In severe cases an outflow obstruction of the right ventricle can develop. This obstruction is caused by accumulation of air forming a bubble and can result in a terminal effect (Palmon et al. 1997). In humans, bolus injections of $3-5 \mathrm{ml} / \mathrm{kg}$ of air have been reported to be fatal, and in dogs the lethal volume of air is $7.5 \mathrm{ml} / \mathrm{kg}$ when injected rapidly (Palmon et al. 1997). Air can be entrained to the venous system through a catheter due to a sub-atmospheric pressure. Using a 14 gauge needle, at a pressure difference of $5 \mathrm{mmHg}$ a flow up to $100 \mathrm{ml}$ air per second is reached (Palmon et al. 1997). If horses can cope with a similar air volume to body weight ratio as humans, it could be extrapolated that for a $500 \mathrm{~kg}$ horse a bolus of $1500-3750 \mathrm{ml}$ of air would be fatal. This volume of air might be sucked into a $14 \mathrm{~g}$ catheter as fast as within 15-25 seconds, but might be altered through catheter clotting and a lower or negative pressure difference when the head position is changed. The exact air volume for horses to become fatal is unknown and translating such data from dogs into the equine does not seem appropriate.

Paradoxical embolism occurs if venous emboli shunt to the arterial side of circulation (van Hulst et al. 2003). This can either happen if the pulmonary filter capacity is exceeded, or if anatomical abnormalities, for example a persistent foramen ovale, are present (Muth and Shank 2000). A study in dogs showed that the amount of air needed to surpass the filtration capability is $0.35 \mathrm{ml} / \mathrm{kg}$ (Adornato et al. 1978). In horses, clinical signs can be seen if an air volume of $0.25 \mathrm{ml} / \mathrm{kg}$ is exceeded (Muir and Hubbell 2010). The pulmonary threshold in these cases reported was clearly exceeded, as air bubbles could be demonstrated ultrasonographically in the left atrium and ventricle of the heart, and no anatomical abnormalities were detected. Therefore these cases have to be considered as paradoxical air embolism with clinical signs of both venous and arterial air embolism.

The clinical symptoms depend on the type of embolism (arterial vs. venous), but also on the rate and amount of air entrained. If air enters the venous system, it immediately affects the heart and subsequently the lung. In the heart air bubbles influence the flow mechanism by either completely blocking cardiac outflow or just by changing flow dynamics of the blood. Occlusion of lung vessels by microbubbles increases the pressure in the pulmonary artery, consequently influencing cardiac output, and leads to tachycardia and arrhythmia (Muth and Shank 2000, van Hulst et al. 2003, Mirski et. al. 2007). The tachycardia seen in these cases is consistent with 
other reported equine (Bradbury et al. 2005, Caporelli et al. 2009, Holbrook et al. 2007, Pellegrini-Masini et al. 2009) and human patients (Thackray et al. 1996, Mirski et al. 2007). The tachyarrhythmia and absent peripheral pulses of the gelding are either a consequence of venous air embolism or might also be a response to the condition by means of cardiovascular centralization and shock, or both. Moreover, air embolism provokes a feeling of suffocation and pain, which may cause tachycardia (Orebaugh 1992). The tachycardia in case 2 might either be caused by the before mentioned mechanisms or by the agitation due to the severe pruritus observed in this case.

Tachypnoe in cases of air embolism is a consequence of decreased oxygen saturation due to the lower blood flow and partial obstruction of the pulmonary vessels (Palmon et al. 1997). This could be an explanation for the tachypnea in both cases. Additionally, if air bubbles become entrapped in the microcirculation of the lung, endothelial cells get injured and due to activation of various vasoactive mediators lung edema can occur (van Hulst et al. 2003). Episodes of lung edema have been reported (Holbrook et al. 2007), however the horses described here never showed any clinical evidence of this condition, although subclinical pulmonary edema cannot be excluded.

If air passes through the lungs because the filter capacity is exceeded, it enters the arterial system. Arterial gas embolism results in distribution of air throughout the whole body. The typical clinical signs like disorientation, seizures and coma are mainly caused by small emboli in the cerebral capillaries and the following ischemia distal to the emboli (van Hulst et al. 2003). Moreover, microbubbles irritate the endothelium of cerebral vessels and this inflammatory response leads to a breakdown of the blood-brain barrier, initiating neurological symptoms (van Hulst et al. 2003).

Obstruction of small cerebral vessels with microbubbles and hence hypoxia of conjunctive brain areas, or hypoxia due to low oxygen saturation and hypoperfusion of the central nervous system is considered to be the cause of the neurological signs (patient disorientation and confusion and, in more severe cases, convulsions, hemiparesis and coma) as seen in human patients (Thackray et al. 1996, Eier and Lock 2003). Besides ischaemia, cerebral edema caused by high arterial pressure in the prestenotic areas can occur (Palmon et al. 1997). Neurological signs have also been described in horses suffering from venous emboli. In the published equine cases horses were violently pacing and kicking, showing hypermetria and moderate ataxia, headpressing, severe pruritus, lack of menace response, blindness, even a grand mal seizure is reported (Bradbury et al. 2005, Caporelli et al. 2009, Holbrook et al. 2007, Pellegrini-Masini et al. 2009). The gelding reported here showed two episodes of focal seizures, consisting of muscle fasciculations, severe tachycardia (200 beats/minute), abnormal head-neck position and severe ataxia. There was never any lack of menace response. The clinical signs faded within 12 hours, as described in human patients (Thackray et al. 1996).

Ischemia probably can occur in any body part following obstruction of capillaries by air bubbles. In these cases probably skin and muscle capillaries. Pruritus as seen in case 2 has been described in other cases with air embolism in the past. The swelling of back muscles the gelding showed is neither reported in human nor in animal cases. The slight increase in creatine kinase can be interpreted as sign of local muscle damage. The most likely explanation would be local ischemia followed by cell damage, caused by microbubbles of air obstructing arterioles. As it is known from humans that air embolism is a painful incident (Orebaugh 1992), it is also possible that the back muscles cramped as a response to pain.

The gold standard of diagnosing air embolism in humans is transesophageal echocardiography. This method is sensitive enough to detect as little as $0.02 \mathrm{ml} / \mathrm{kg}$ of air when administered as a bolus (Mirski et al. 2007). It is used in human medicine as a monitoring tool during cerebral and cardiac surgery. Less invasive, and only slightly inferior, is the precordial Doppler ultrasound, which still has a sensitivity to detect $0.05 \mathrm{ml} / \mathrm{kg}$ of air in the vascular system (Mirski et al. 2007).

So far in all reported equine cases venous air embolism was suspected on the basis of history of an open venous catheter site (Bradbury et al. 2005, Caporelli et al. 2009, Holbrook et al. 2007, Pellegrini-Masini et al. 2009). Both horses in this report were also found with a dislodged catheter cap/extension set. Transthoracic ultrasound of the heart, pulmonary vessels and aorta confirmed the diagnosis of air embolism by showing air bubbles not only in the right but also in the left atrium and ventricle. In order to exclude anatomical abnormalities which could explain this right-left shunt, a bubble study was performed. These studies are used in humane medicine for diagnosing patent foramen ovale (Meier and Lock 2003), coronary artery disease (Kaul 2001) and pulmonary arteriovenous shunting (Chang et al. 1999). As no abnormality was found in these two cases, the presence of air bubbles on the left side of the heart was explained by the amount of air exceeding the pulmonary threshold and leading to a paradoxical air embolism.

Once venous air embolism is suspected further air entry should be prevented and the horse treated as an emergency case. Initial treatment consisted of cardiovascular support by rapid volume expansion (Muth and Shank 2000). Treatment with colloid solutions does not only support the cardiovascular system but might also help to prevent cerebral edema (Muth and Shank 2000). In case of air obstruction of the right ventricle evacuation of air from the right ventricle with a right atrial catheter is described in humans (Palmon et al. 1997) but not in horses. It should be kept in mind that with proper equipment this treatment would be applicable in horses, but was not deemed necessary in these cases. Oxygen support has been proven to be essential in humans. This does not only improve tissue oxygenation but also seems to reduce the volume of emboli (Mirski et al. 2007). Although oxygen treatment has not been mentioned as an additional treatment for horses, the gelding received 100\% oxygen intranasally. Considering the reported benefits in humans and the low $\mathrm{O}_{2}$ saturation it was considered that supportive oxygen treatment might improve the condition of the horse. This point might be a matter of discussion because intranasal oxygen delivery might not result in an increased oxygenation in horses with a $\mathrm{PaO}_{2}$ over $60 \mathrm{mmHg}$, however, nasopharyngeal oxygen delivery in normal horses increased the inspired fraction of oxygen $\left(\mathrm{FlO}_{2}\right.$ ) as well as the $\mathrm{PaO}_{2}$ (Wilson et all 2006). 
Because case 1 suffering from cardiac arrhythmia lidocaine was administered. According to recent literature reviews, there are also indications that lidocaine might be beneficial in cases of gas embolism (Muth and Shank 2000, van Hulst et al. 2003). It decreased the elevated intracranial pressure due to air embolism in animal studies. The use of lidocaine might therefore have been of additional benefit to prevent further neurological symptoms in this case, even if the exact mechanisms of lidocaine decreasing intracranial pressure remain unclear.

To terminate the seizure attacks a benzodiazepine was administered. Case 1 initially responded well, but then showed further seizures episodes half an hour later. In humans with cerebral gas embolism, it is known that they often do not respond to benzodiazepines. Barbiturates reduce cerebral oxygen consumption and release of catecholamines. Therefore barbiturates might be beneficial for cases of cerebral air embolism (Muth and Shank 2000, van Hulst et al. 2003). Phenobarbitone was used in this case because it is a wellknown drug to treat focal seizures in horses (Mayhew 2008) and was of benefit in a case of air embolism in the past (Pellegrini-Masini et al. 2009). After administering phenobarbitone, the gelding did not show further signs of seizures.

Case 1 was also treated with antimicrobials, however the necessity could be debated. It was administered in order to prevent secondary bacterial infection of compromised lung tissue. Case 2 already received antibiotics for other reasons and the treatment was continued as in other reported cases (Bradbury et al. 2005, Caporelli et al. 2009, Holbrook et al. 2007, Pellegrini-Masini et al. 2009). Case 2 was additionally sedated with a short acting $\alpha 2$ agonist to calm the horse down as it was very agitated in response to the severe pruritus. Alpha2 agonists raise CSF pressure and cause vasoconstriction which could potentially be a disadvantage regarding blood flow to already compromised tissue. The horse did not show any other signs of neurological disease than pruritis at this point and calming it down seemed important to prevent it from further kicking the already bleeding surgical site.

The mare's catheter was not immediately taken out, only the broken extension set was changed. The horse continued to receive its medication via this catheter and developed a perithrombophlebitis, which was successfully managed. It might be advisable to take out a catheter which led to air embolism immediately to prevent further problems.

As reported in these cases, air embolism in the horse is a manageable situation as long as there is immediate support of the horse's condition. The diagnosis of air embolism can be confirmed with transthoracic echocardography, which seems to be a sensitive and practical way for detecting intracardiac air, although more experience with this method is necessary When detected in the left side of the heart, it can be concluded that the lungs were unable to reabsorb all air and paradoxical embolism might occur, with further clinical signs to be expected. Most of the symptoms seem to occur immediately but some, as the neurological signs in case 1 can be delayed. Air embolism is a problematic condition with variable clinical signs depending on the rate and amount of air entrapped. According to the human and veterinary literature no long term effects are expected when responding to treatment (Bradbury et al. 2005, Caporelli et al. 2009, Holbrook et al. 2007, Pellegrini-Masini et al. 2009), (Thackray et al. 1996). Although clinical signs are manageable, prevention of venous air embolism still remains most important.

\section{Manufacturers`addresses}

1 Laboratoire Pharmaceutique Vygon, France

2 Schiller AG, Baar, Switzerland

3 Braun AG, Germany

4 Gespag, Linz, Austria

5 Fresenius Kabi, Graz, Austria

6 Gebro Pharma GmbH, Fieberbrunn, Austria

7 GE Medical Systems, Solingen, Germany

8 Kwizda Pharma, Vienna, Austria

9 Nycomed Austria, Linz, Austria

10 Desitin Hamburg, Germany

11 Boehringer Ingelheim, Ingelheim, Germany

12 Albrecht, Aulendorf, Germany

13 Animedica, Senden, Germany

14 Vetoquinol, Ravensburg, Germany

15 Mila International, USA

16 Esaote, Köln, Germany

17 Rebopharm, Bocholt, Germany

\section{References}

Adornato D. C., Gildenberg P. L., Ferrario C. M., Smart J., Frost E. A. M. (1978) Pathophysiology of Intravenous Air Embolism in Dogs. Anesthesiology 49, 120-127

Bradbury L. A., Archer D. C., Dugdale A. H. A., Senior J. M., Edwards G. B. (2005) Suspected venous air embolism in a horse. Vet. Rec. 156, 109-111

Caporelli F., McGowan C. M., Tulamo R. M. (2009) Suspected venous air embolism in a Finnhorse. Equine Vet. Educ. 21, 85-88

Chang R.-K. R., Alejos J. C., Atkinson D., Jensen R., Drant S., Galindo A., Laks H. (1999) Bubble contrast echocardiography in detecting pulmonary arteriovenous shunting in children with univentricular heart after cavopulmonary anastomosis. J. Am. Coll. Cardiol. 33, 2052-2058

Holbrook T. C., Dechant J. E., Crowson C. L. (2007) Suspected air embolism associated with post-anesthetic pulmonary edema and neurologic sequelae in a horse. Vet. Anaest. Analg. 34, 217-222

Kaul S. (2001) Myocardial contrast echocardiography: Basic principles. Progress in Cardiovascular Diseases 44, 1-11

Marek A. Mirski, M. D., Abhiiji Vijay Lala M. D., Lunei Fitzsimmons M. D., Thomas J. K., Toung M. D. (2007) Diagnosis and Treatment of Vascular Air Embolism. Anesthesiology 106, 164-177

Mayhew I. (2008) Seizures and Epilepsy. In: Large Animal Neurology, 2nd Edition edn., Wiley-Blackwell Publishing. p 86

Meier B., Lock J. E. (2003) Contemporary Management of Patent Foramen Ovale. Circulation 107, 5-9

Muir W., Hubbell J. (2010) Complication: introduction, mantainance and recovery phases of anesthesia. In: Equine Anesthesia, Second edn., Saunders. pp 419-443

Muth C. M., Shank E. S. (2000) Gas Embolism. New Engl. J. Med. 342, 476-482

Nanthakumar K., Graham A. T., Robinson T. I., Grande P., Pugash R. A., Clarke J. A., Hutchison S. J., Mandzia J. L., Hyland R. H., Faughnan M. E. (2001) Contrast echocardiography for detection of pulmonary arteriovenous malformations. Am. Heart J. 141, 243-246

Orebaugh S. L. (1992) Venous air embolism: Clinical and experimental considerations. Crit. Care Med. 20, $1169-1177$

Palmon S. C., Moore L. E., Lundberg J., Toung T. (1997) Venous air embolism: A review. J. Clin. Anesth. 9, 251-257 
Pasternak J. J., Lanier W. L. (2017) Diseases affecting the brain. Stoelting's Anesthesia and Co-Existing Disease E-Book, ed. Hines R L., Marschall K. 7th Ed. , Elsevier, 275-277

Pellegrini-Masini A., Hurtado I. R., Stewart A. J., Divers T. J. (2009) Suspected venous air embolism in three horses. Equine Vet. Educ. 21, 79-84

Thackray N. M., Murphy P. M., McLean R. F., delacy J. L. (1996) Venous air embolism accompanied by echocardiographic evidence of transpulmonary air passage. Crit. Care Med. 24, 359-361 van Hulst R. A., Klein J., Lachmann B. (2003) Gas embolism pathophysiology and treatment. Clin. Physiol. Funct. Imag. 23, 237 246

Wilson D. V., Schott II H. C., Robinson N. E., Berney C. E., Eberhart S. W. (2006) Response to nasopharyngeal oxygen administration in horses with lung disease. Equine Vet. J. 38, 219-223 